\title{
An alternative theoretical approach to describe planetary systems through a Schrödinger-type diffusion equation
}

\author{
M. de Oliveira Neto ${ }^{1}$, L. A. Maia ${ }^{2}$ and S. Carneiro ${ }^{3}$ \\ ${ }^{1}$ Instituto de Química, Universidade de Brasília, 70910-900, Brasília, Brazil \\ ${ }^{2}$ Departamento de Matemática, Universidade de Brasília, 70910-900, Brasília, Brazil \\ ${ }^{3}$ Instituto de Física, Universidade Federal da Bahia, 40210-340, Salvador, BA, Brazil
}

\begin{abstract}
In the present work we show that planetary mean distances can be calculated with the help of a Schrödinger-type diffusion equation. The obtained results are shown to agree with the observed orbits of all the planets and of the asteroid belt in the solar system, with only three empty states. Furthermore, the equation solutions predict a fundamental orbit at 0.05 AU from solar-type stars, a result confirmed by recent discoveries. In contrast to other similar approaches previously presented in the literature, we take into account the flatness of the solar system, by considering the flat solutions of the Schrödinger-type equation. The model has just one input parameter, given by the mean distance of Mercury.
\end{abstract}

\section{INTRODUCTION}

In recent years, some authors [1]-[10] have suggested a quantum-like approach to calculate the planetary orbits in our solar system, which has led to several impressive results. Besides obtaining the observed orbits of all the planets and of the asteroid belt, such models have made predictions subsequently confirmed by observations.

For example, the existence of asteroids orbiting between Uranus and Neptune, with orbit radii around 24.77 AU [1], was confirmed by recent discoveries [1]. Furthermore, the prediction of two intramercurial orbits with radii around $0.05 \mathrm{AU}$ and $0.18 \mathrm{AU}$ 4, 7], absents of our solar system, has been verified in several extra-solar planetary systems during the last years [12].

In spite of these and others positive results, these approaches seem to be very speculative, for the quantization of macroscopic systems is something outside the scope of our known physics. Nevertheless, some possible origins for such effects have been outlined, some of them on the basis of quite orthodox theories.

A large scale quantization can be based, for instance, on an extension of the ordinary commutation rules, in order to recover the equivalence principle in the context of quantum mechanics [7, 13]. The general commutation rules derived in this way predict two scales of quantization: the usual microscopic one, and the scale in which the gravitational interactions are dominant. The presence of quantization at so different scales can be related as well to self-similarity concepts 2]. And it is also characteristic of fractal space-time approaches [3], in which the particle trajectories are non-differentiable at very short and very large scales.

The concept of non-differentiability of space-time at short scales has been used by some authors in trying to derive the Schrödinger equation in the context of a classical framework. For example, in the stochastic mechanics of Nelson [14], the Schrödinger equation is obtained as a classical diffusion equation, with help of the hypothesis that any particle in the empty space, under the influence of any interaction field, is also subject to a universal
Brownian motion without viscosity. Under this hypothesis, a Schrödinger-type equation follows from classical dynamics, with the Planck constant being related to a diffusion coefficient.

The main problem of this kind of derivation of the Schrödinger equation (as well as of other descriptions based on diffusion processes or fluid dynamics [15]) is looking for a convincing physical origin for that universal Brownian motion, although some possibilities have been outlined, based for instance on quantum fluctuations on cosmic scale [16], or on the quantum nature of space-time in quantum gravity theories [17].

Nevertheless, the important point in Nelson's work is that a diffusion process can be described in terms of a Schrödinger-type equation. In this context, the possibility of describing a classical process like the formation of solar system in terms of quantum mechanics can be seriously considered. The use of such an approach is also suggested by the chaotic behaviour of the solar system during its formation and evolution [18]-[21], which implies the non-differentiability of trajectories for large time scales [5].

Despite all these conjectures, the physical principles behind the quantization of large structures cannot be considered completely understood. In the present work, our aim is not to solve this problem, but just to explore a little more the results we can obtain from a quantum-like approach. Our goal is to introduce a new ingredient not considered up to now: the flatness of the solar system. For this purpose, we will look for flat solutions of the Schrödinger-type equation, in contrast to the spherical solutions usually considered in the previous papers.

\section{PRELIMINARY REMARKS}

Applying a Bohr-like "quantization" rule to the orbital angular momentum of a planet in circular orbit around the Sun, $L=m v r=n g^{*} / 2 \pi$, and using the Newtonian law for the orbital velocity, $v^{2}=G M / r$, one of the authors [1] has derived the following expression for the radii 
of the orbits:

$$
r=\frac{n^{2} g^{* 2}}{4 \pi^{2} G M m^{2}},
$$

where $n$ is an integer, $G$ is the gravitational constant, $M$ is the solar mass, $m$ is the planet mass and $g^{*}$ stands for a quantum of action, playing the role of a re-scaled Planck constant.

In the case $n=1$, equalizing (11) to the observed value of the Mercury orbital radius, using the observed values for $G$ and $M$, and taking $m=2.1 \times 10^{26} \mathrm{~kg}$ (the average mass of the planets of the solar system), we find $g^{*}=3.6 \times 10^{42}$ J.s. This value coincides to a re-scaled quantum of action derived on the basis of self-similarity considerations [2].

Now, equation (11) can be written as $r=n^{2} r_{1}$, where $r_{1}$ is the Mercury orbital radius. Taking for $n$ the next integer values, we can obtain a sequence of values that assures very well the observed values of orbital radii in our solar system [1]. For $n=2$ we obtain the orbit of Mars; for $n=3$ the orbit of Camilla (an asteroid located in the outer region of the asteroid belt between Mars and Jupiter); the values $n=4$ and $n=5$ give the orbits of Jupiter and Saturn, respectively; for $n=6$ we have the orbital radius of Chiron (an asteroid between Saturn and Uranus); and, for $n=7,9$ and 10, the orbits of Uranus, Neptune and Pluto follow, in this order. The deviations from the observed values are $19 \%$ for Jupiter, $4 \%$ for Neptune and less than $2 \%$ in the other cases.

The value $n=8$ predicts an orbit between Uranus and Neptune, with radius 24.8 AU. In reference [1], submitted for publication in October 1994, this orbit was considered empty. Surprisingly enough, the value above is in very good agreement with subsequent discoveries of two asteroids located precisely at this radial position, named 1993 HA 2 and 1995 DW 2 [1]. These discoveries were published in 1995, that is, one year after the paper submission, showing the predict power of this kind of model.

The most important drawback of the model is, as already noted by the reader, the absence of Venus and Earth in the above sequence. To include these planets, and also the asteroid Vesta, located in the inner region of the asteroid belt, it has been taken into account an ad hoc second quantum number $n^{\prime}$, running from 0 to $n$ [1], such that relation (11) is generalized to

$$
r=\frac{\left(n^{2}+n^{\prime 2}\right) g^{* 2}}{8 \pi^{2} G M m^{2}}
$$

For $n=n^{\prime}$ we recover the original relation and results.

Another possibility was provided by Agnese and Festa 7], taking for $n=1$, instead of the Mercury radius, the value $r_{1}=0.04 \mathrm{AU}$, which corresponds to $g^{*}=1.2 \times$ $10^{42}$ J.s. Now, for $n$ running from 3 to 6 , we obtain the orbital radius of Mercury, Venus, Earth and Mars; for $n=8$ we have the asteroid Ceres; and, for $n=11$, 15, 21, 26 and 30, the orbital radius of Jupiter, Saturn,
Uranus, Neptune and Pluto follow, in agreement with the observed values. The problem here, as we can see, is that we fall into a lot of empty orbital positions, that is, radius values predicted by the formula $r=n^{2} r_{1}$, but not occupied by any observed body, particularly for large values of $n$. Nevertheless, the prediction of a fundamental radius at $0.04 \mathrm{AU}$ would be shown an important result.

Another interesting contribution by Agnese and Festa is the introduction of the re-scaled fine structure constant $\alpha_{g}=2 \pi G M m / g^{*} c$, where $c$ is the light velocity. In this way one can rewrite equation (11) as

$$
r=\frac{n^{2} G M}{\alpha_{g}^{2} c^{2}} .
$$

Using for $m$ the average mass of planets of our solar system and for $g^{*}$ the value obtained above, we obtain for $\alpha_{g}$ the value used by Agnese and Festa, $\alpha_{g}^{-1}=2.1 \times 10^{3}$.

This quantum approach also works for other, recently discovered, planetary systems, as shown by Nottale [4, 6] and also by Agnese and Festa [8, [9]. In Nottale's approach the periods of planets revolution are quantized as

$$
T=2 \pi G M n^{3} / \omega_{0}^{3},
$$

where $\omega_{0}=144 \mathrm{~km} / \mathrm{s}$ is a characteristic velocity. Using $T=2 \pi r / v, v^{2}=G M / r$ and $r$ given by (3), we derive $\omega_{0}=\alpha_{g} c$. Using $\omega_{0}=144 \mathrm{~km} / \mathrm{s}$, we obtain $\alpha_{g}^{-1}=$ $2.1 \times 10^{3}$, which again is in accordance with the value obtained by Agnesa and Festa.

Therefore, Nottale also predicts a fundamental radius given by $r_{1} \approx 0.04 \mathrm{AU}$. Several extra solar planets recently discovered lie at this distance from their star [12]. This agreement between a fundamental radius derived years ago and subsequent observations shows once again the predict power of such models.

Some of the above results come from a semi-classical treatment of the problem, based essentially on the application of a Bohr-like quantization condition. In Nottale's works we can find a more rigorous approach, based on a Schrödinger-type wave equation. In this way he can accommodate all the planets and the asteroid belt of our solar system, with only a few empty orbits [3]. To achieve this goal he treats the inner and outer systems separately, with two distinct values of $\omega_{0}$.

In the next section, we propose to show that flat solutions of the Schrödinger-type equation can furnish important information on the mean planetary radii. Our aim is to enrich the previous studies, including a new ingredient not considered up to now: the flatness of the solar system and of the original disk from which it was originated.

\section{THE FLAT SOLUTIONS}

In this section we present the mathematical results obtained from the solution of a Schrödinger-type equation involving an attractive central field. As already 
mentioned, the orbits of planets and asteroids will be considered approximately in the same plane, and the 3dimensional equation will be solved taking $z=0$.

Let us consider a body of mass $m$ moving around another body of mass $M$, under an attractive central field with potential $V(r)$, where $r$ is the axial distance in polar coordinates. The Schrödinger-type equation is given then by

$$
-\frac{g^{* 2}}{2 \mu}\left(\frac{\partial^{2} \psi}{\partial r^{2}}+\frac{1}{r} \frac{\partial \psi}{\partial r}+\frac{1}{r^{2}} \frac{\partial^{2} \psi}{\partial \theta^{2}}\right)+V(r) \psi=E \psi .
$$

The term in parenthesis is $\Delta \psi$, where $\Delta$ is the Laplace operator in polar coordinates $(r, \theta)$, which is applied to the wavefunction $\psi$ of the body of mass $m$. The parameter $E$ stands for the total energy of the system; $\mu$ is the reduced mass $m M /(m+M)$; and $g^{*}$ is a constant, as previously described.

As long as the potential $V$ is a function of the radial variable only, we may look for a solution using separation of variables,

$$
\psi(r, \theta)=f(r) \Theta(\theta)
$$

Replacing (6) in (5) and dividing by $f(r) \Theta(\theta)$, we get

$$
\begin{gathered}
\frac{g^{* 2}}{2 \mu} \frac{1}{f(r)}\left[\frac{d^{2} f(r)}{d r^{2}}+\frac{1}{r} \frac{d f(r)}{d r}\right]+ \\
+\frac{g^{* 2}}{2 \mu r^{2}} \frac{1}{\Theta(\theta)} \frac{d^{2} \Theta(\theta)}{d \theta^{2}}+E-V(r)=0,
\end{gathered}
$$

or, equivalently,

$$
\begin{array}{r}
\frac{1}{\Theta(\theta)} \frac{d^{2} \Theta(\theta)}{d \theta^{2}}=-\frac{r^{2}}{f(r)}\left[\frac{d^{2} f(r)}{d r^{2}}+\frac{1}{r} \frac{d f(r)}{d r}\right]- \\
-\frac{2 \mu r^{2}}{g^{* 2}}[E-V(r)]
\end{array}
$$

Since the term on the left hand side depends only on $\theta$ and the term on the right hand side depends only on $r$, both terms must be equal to a constant which we denote by $-\ell^{2}$. Therefore, we obtain two ordinary differential equations:

$$
\Theta^{\prime \prime}(\theta)=-\ell^{2} \Theta(\theta)
$$

and

$$
f^{\prime \prime}(r)+\frac{1}{r} f^{\prime}(r)+\left\{-\frac{\ell^{2}}{r^{2}}+\frac{2 \mu}{g^{* 2}}[E-V(r)]\right\} f(r)=0 .
$$

Equation (9) has a solution

$$
\Theta(\theta)=e^{i \ell \theta} .
$$

If we assume the boundary condition

$$
\Theta(0)=\Theta(2 \pi),
$$

we end up with $|\ell|=0,1,2,3 \ldots ; \ell$ is an integer.
Now we apply a rescaling in (10) by

$$
\rho=2 \beta r, \quad \beta>0,
$$

where $\beta^{2}=-2 \mu E / g^{* 2}$. Furthermore we define

$$
n=\frac{\mu G M m}{g^{* 2} \beta},
$$

and, by using $V(r)=-G M m / r$, equation (10) becomes

$$
\tilde{f}^{\prime}(\rho)+\frac{1}{\rho} \tilde{f}^{\prime}(\rho)+\left(-\frac{1}{4}-\frac{\ell^{2}}{\rho^{2}}+\frac{n}{\rho}\right) \tilde{f}(\rho)=0 .
$$

If we consider $\tilde{f}(\rho)=\frac{1}{\sqrt{\rho}} u(\rho)$ in (15) we obtain

$$
u^{\prime \prime}(\rho)+\left[-\frac{1}{4}+\frac{n}{\rho}-\frac{\left(\ell^{2}-1 / 4\right)}{\rho^{2}}\right] u(\rho)=0 .
$$

For this equation, $\rho=0$ is a regular singular point and $\rho=\infty$ is an irregular singular point. Equation (16) is a confluent hypergeometric equation [22], referred as Whittaker's equation 23]. This equation has a regular solution given by a hypergeometric series which converges if, and only if,

$$
n=|\ell| \pm \frac{1}{2} \pm k, \quad k=0,1,2,3 \ldots
$$

By definition, $n \geq 0$. Assuming that the solution $u(\rho)$ satisfies the boundary condition

$$
\lim _{\rho \rightarrow+\infty} u(\rho)=0
$$

and as $u(0)$ must be finite, we have

$$
n=|\ell|+\frac{1}{2}+k, \quad k=0,1,2,3 \ldots
$$

So far we have the conditions

$$
\begin{aligned}
& |\ell|=0,1,2,3 \ldots \\
& n=|\ell|+\frac{1}{2}, \quad|\ell|+\frac{3}{2}, \quad|\ell|+\frac{5}{2} \ldots
\end{aligned}
$$

They can be regrouped in the form

$$
\begin{aligned}
n & =\frac{1}{2}, \frac{3}{2}, \frac{5}{2}, \frac{7}{2} \ldots \\
\ell & =0,1,2, \ldots, n-\frac{1}{2} .
\end{aligned}
$$

For each pair $n \ell$, the solutions $u_{n \ell}$ were obtained using Maple V Release 4 Software (Waterloo Maple Inc.). As long as we obtain $u_{n \ell}(\rho)$, we may find the solution $f_{n \ell}(r)$ of the original equation (10). Once we know the collection of functions $f_{n \ell}(r)$ and $\Theta_{\ell}(r)$, their products generate the normalized solutions $\psi_{n \ell}$ of the Schrödingertype equation. 
Now we define

$$
P_{n \ell}(r) d r=\int_{0}^{2 \pi} \psi_{n \ell}^{*} \psi_{n \ell} r d r d \theta
$$

which is equivalent to

$$
P_{n \ell}(r) d r=r[f(r)]^{2} d r .
$$

As in the case of atomic wavefunctions, the mean radius is obtained by

$$
r_{n \ell}=\int_{0}^{\infty} r P_{n \ell}(r) d r=\int_{0}^{\infty} \int_{0}^{2 \pi}\left(\psi_{n \ell}^{*} r \psi_{n \ell}\right) r d r d \theta,
$$

which is equivalent to

$$
r_{n \ell}=\int_{0}^{\infty}[r f(r)]^{2} d r .
$$

In this context, we now return to the rescaling factor $\beta$ and consider the mean distance of Mercury as $r_{\frac{3}{2} 0}$. (If we associate Mercury with the first state $r_{\frac{1}{2} 0}$, all results are inconsistent with the observed mean planetary distances. The orbits of Venus, Earth, Mars and Jupiter, for example, definitely cannot be derived.) For $n=3 / 2$ and $l=0$, we obtain for Eq. (16) the solution

$$
\frac{1}{\sqrt{\rho}} u(\rho)=c_{1}\left(-e^{-\frac{1}{2} \rho}+e^{-\frac{1}{2} \rho} \rho\right)
$$

and so $f(r)=c_{1} e^{-\beta r}(2 \beta r-1)$. Since $\int_{0}^{\infty} r[f(r)]^{2} d r=1$ we have

$$
c_{1}=\sqrt{\frac{4}{3}} \beta
$$

From Eq. (25) we then get

$$
r_{\frac{3}{2} 0}=\frac{7}{3} \frac{1}{\beta}=0.387 A U
$$

where we have used the observed mean distance of Mercury. So, we obtain

$$
\beta=\beta_{\frac{3}{2}}=\frac{1}{0.387} \frac{7}{3}=9.04 /(3 / 2) A U^{-1} .
$$

Therefore, since $\beta$ is proportional to $1 / n$ (see Eq. (14)), for each value of $n$ we will consider

$$
\beta_{n}=9.04(1 / n) A U^{-1} .
$$

Its important to remark that the orbital radius of Mercury will be the only input parameter used in this model.

\section{RESULTS AND DISCUSSIONS}

We denote by the triple $(n, \ell, r)$ the resulting numbers $n, \ell$, and the corresponding mean planetary radius $r_{n \ell}$ (in
$\mathrm{AU})$, calculated from equation (25). The first triple found $(1 / 2,0,0.055)$ does not correspond to any observed orbit in our solar system. As previously mentioned, Nottale [4] also found this radius as the fundamental level in his quantization law, and it was also obtained by Agnese and Festa as the first state in their quantization condition [7]. As remarked in these two papers, the first extra-solar planet discovered around the solar-type 51 Pegasus lies precisely at $0.05 \mathrm{AU}$ from its star. Other subsequently discovered companions of solar-type stars, which also fall around $0.05 \mathrm{AU}$, confirm this result 12 ].

The following two states $(3 / 2,0,0.387)$ and $(3 / 2,1,0.332)$ are associated with the orbit of Mercury. The triples $(5 / 2,0,1.05)$ and $(5 / 2,1,0.995)$ have very similar values for $r$ which are equal to the mean planetary distance of Earth. The triple corresponding to Venus is $(5 / 2,2,0.83)$.

The set of triples $(7 / 2,0,2.04),(7 / 2,1,1.99)$ and $(7 / 2,2,1.82)$ does not correspond to any observed planet in this region of the solar system, but could be associated with the asteroid Hungarias, at $1.94 \mathrm{AU}$. This radius value was also predicted by Nottale in his quantization law 3] and nearly corresponds to the radius found for a companion of the solar-type star $47 \mathrm{UMa}$ B, lying at 2.12 $\mathrm{AU}$ from this star (see 4, 7] and references therein).

The state $(7 / 2,3,1.54)$ clearly stands for the orbit of Mars. The results obtained for $n=9 / 2$ are $(9 / 2,0,3.37)$, $(9 / 2,1,3.32),(9 / 2,2,3.15),(9 / 2,3,2.88)$ and $(9 / 2,4,2.49)$. They are in a very good correspondence with the asteroid belt in the solar system. The lower values $2.88 \mathrm{AU}$ and 2.49 AU correspond to the mean distances observed for the Ceres group (2.64 AU), the central peak of the belt, and for Vesta (2.36 AU), which delimits its interior ring. The upper values 3.32 AU and 3.37 AU correspond to the mean distance observed for Camilla (3.48 AU), which delimits the exterior ring. The intermediate value 3.15 $\mathrm{AU}$ has a precise correspondence with the Hygeia group (3.16 AU), the main peak of the asteroid belt.

For larger values of $n$, a great number of states is found. Nevertheless (as pointed out by Nottale et al. [5] to explain the circularity of the orbits in the solar system), a great number of states occupying the same region of space, most of them with large eccentricities, leads to a strong chaos and to the crossing of orbits, which on large time scales would generate the condensation of states on the observed, approximately circular orbits. Therefore, for $n>9 / 2$ we will consider only the states with rotational symmetry, that is, with $l=0$ (see Eq. (11)).

In this way, the states $(11 / 2,0,5.03)$ and $(15 / 2,0,9.34)$ can be associated with the orbits of Jupiter and Saturn, respectively. The states $(17 / 2,0,12.0)$ and $(19 / 2,0,15.0)$ give mean values around the orbit of the asteroid Chiron, distant 13.7 AU from the Sun. The state $(21 / 2,0,18.3)$ can be associated with the orbit of Uranus, while the state $(25 / 2,0,25.9)$ has a mean distance close to the orbital radii of the already referred asteroids 1993 HA 2 and 1995 DW 2. The next state, (27/2,0,30.2), clearly stands for the orbit of Neptune and, finally, the state 
$(31 / 2,0,39.9)$ can easily be associated with the orbit of Pluto.

As one can see, all the planets in the solar system (as well as the asteroid belt) are fitted by the model. Apart the fundamental radius $0.055 \mathrm{AU}$ (which should be ruled out for thermodynamical reasons [5]), we have only three empty orbits, corresponding to the states $(13 / 2,0,7.02)$, $(23 / 2,0,21.9)$ and $(29 / 2,0,34.9)$. In what concerns the occupied states, the deviations from the observed orbital radii are $15 \%$ for Venus, $10 \%$ for Chiron, $6 \%$ for Ceres and Vesta, $5 \%$ for Uranus and the asteroids HA2 and DW2, $3 \%$ for Jupiter, Camilla and Hungarias, 2\% for Saturn, $1 \%$ for Mars and Pluto, and 0.5\% for Earth, Neptune and Hygeia.

Beyond the orbit of Pluto, we still have the states $(33 / 2,0,45.2)$ and $(35 / 2,0,50.8)$, which could correspond to other mass peaks in the Kuiper belt, a group of about 60 trans-Neptunian objects orbiting up to $50 \mathrm{AU}$ from the Sun 24, 25]. [After the completion of this work, it was announced the discovery of Quaoar, the biggest asteroid discovered up to now in the Kuiper belt 26]. Its stable orbit has mean radius around $42 \mathrm{AU}$, which may correspond either to the state $(31 / 2,0,39.9)$, associated to Pluto's peak, or to the state $(33 / 2,0,45.2)$. The relative deviations are $5 \%$ and $8 \%$, respectively.]

\section{CONCLUSIONS}

The set of obtained results in calculating planetary orbits in the present work, as well as those obtained by other authors, are enough to encourage further studies. In all these theoretical approaches, the existence of a fundamental radius around $0.05 \mathrm{AU}$ is predicted, and several planets have been recently discovered orbiting at this radius in extra-solar systems. Furthermore, it was predicted the existence of asteroids between Uranus and Neptune [1], a prediction confirmed by observations.

The Scrödinger-type model presented in this work seems to describe the observed mean distances from Mercury until the asteroid belt. For the outer system we have considered only states with rotational symmetry, obtaining good results as well, with only three empty states. All the results were obtained with just one input parameter, namely the orbital radius of Mercury.

The reader may be asking how a set of planets and asteroids with so different masses can be described by just one equation. In other words, why do we need only one input parameter (the value of $\beta$ for Mercury), if equation (5) depends explicitly on the reduced mass $\mu$ ?

The answer may reside on the fact that our
Schrödinger-type equation is not a genuine quantum equation, but describes a kind of diffusion process. In this context, the re-scaled "Planck constant" present in (5) is related to the diffusion coefficient $\mathcal{D}$ by $g^{*}=2 m \mathcal{D}$ [5, 14], where $m$ is the mass under diffusion. Furthermore, for any planet or asteroid gravitating around the Sun, the reduced mass $\mu$ coincides with its mass $m$. Therefore, remembering that $V(r)$ and $E$ are also proportional to $m$, it is easy to verify that (5), in fact, does not depend on $m$.

Another way of verifying this result is to look at equation (14). Using $\mu=m$ and $g^{*}=2 m \mathcal{D}$, we obtain $\beta=G M /\left(4 \mathcal{D}^{2} n\right)$. So, for a given diffusion coefficient and a given stellar mass, the parameter $\beta$ depends just on $n$, as assumed in Eq. (30).

As a last remark, let us point out an observable difference between the flat model presented here and spherical models. In the approaches by Nottale et al [5] and by Agnese and Festa [7], there is a predicted orbit at 0.18 AU from the Sun, corresponding to $n=2$ (remember that, in their models, Mercury corresponds to $n=3$ ). This orbit is also predicted through the Bohr-like model presented in [1], corresponding to the quantum numbers $n=1, n^{\prime}=0$ (see Eq. (21)). As a little planet orbiting at the fundamental radius $0.05 \mathrm{AU}$ is ruled out by thermodynamical constraints [5], a body at $0.18 \mathrm{AU}$ would be the only new body predicted to orbit near the Sun.

As we have seen in the previous section, such an orbit does not exist in the present model. It is excluded by the flatness of our solutions. Of course, this does not mean that it could not be observed in extra-solar systems. Actually, orbits with radius around this value were already observed [12]. This fact could suggest that, in contrast to our solar system, the corresponding planetary systems are not flat, which could indicate that they were originated in a different way.

\section{Acknowledgments}

We gratefully acknowledge the support and encouragement from Kleber C. Mundim (UnB) and Marcelo M. Moret (UEFS). We would like to thank Naelton Mendes de Araujo ( Sistema Telebras-Embratel) for sending astronomical data about the discovery of the asteroids HA2 and DW2. Also Ricardo Fragelli (UnB) for the extraordinary support on writing Maple programs for the solution of the Schrödinger-type equation. Finally ours thanks to Maristela Rocha (UnB) and Hans-Peter Leeb for reading and providing helpful suggestions on the manuscript. S.C. was partially supported by CNPq, Brazil.
[1] M. Oliveira Neto, Ciência e Cultura (Journal of the Brazilian Association for the Advancement of Science), 48 (1996) 166.

[2] S. Carneiro, Found. Phys. Lett., 11 (1998) 95.
[3] L. Nottale, Fractal space-time and microphysics: towards a theory of scale relativity (World Scientific, Singapore), 1993, p. 311.

[4] L. Nottale, A\&A, 315 (1996) L9. 
[5] L. Nottale, G. Schumacher, and J. Gay, A\&A, 322 (1997) 1018.

[6] L. Nottale, G. Schumacher, and E.T. Lefèvre, A\&A 361 (2000) 379 .

[7] A. G. Agnese and R. Festa, Phys. Lett. A, 227 (1997) 165.

[8] A. G. Agnese and R. Festa, Hadronic J., 21 (1998) 237.

[9] A. G. Agnese and R. Festa, Discretizing u-Andromedae planetary system, astro-ph/9910534, v2, 1999.

[10] H. Arp, Seeing Red: Redshifts, Cosmology and Academic Science (Apeiron, Montreal), 1998, p. 219.

[11] Minor Planet Circular, 25441 (07/12/95) and 25184 (05/14/95), International Astronomical Union.

[12] J. Schneider, Extra-solar planets catalog, http://www.obspm.fr/encycl/catalog.html

[13] D. M. Greenberger, Found. Phys., 13 (1983) 903.

[14] E. Nelson, Phys. Rev., 150 (1966) 1079.

[15] M. S. El Naschie, E. Rossler, and I. Prigogine, Quantum Mechanics, Diffusion and Chaotic Fractals (Perga- mon Press, Oxford), 1995.

[16] B. G. Sidharth, Chaos, Solitons and Fractals, 15 (2003) 25.

[17] S. Bergia, F. Cannata, and A. Pasini, Phys. Lett. A 137 (1989) 21, and references therein.

[18] J. G. Hills, Nature, 225 (1970) 840.

[19] M. Hénon and C. Heiles, Astrophys. J., 69 (1964) 73.

[20] J. Laskar, Nature, 338 (1989) 237.

[21] G. Sussman and J. Wisdom, Science, 257 (1992) 56.

[22] E. L. Ince, Ordinary Differential Equations (Dover Publications Inc., New York), 1956, pp. 184 and 464.

[23] A. D. Polyanin and V. F. Zaitsev, Handbook of exact solutions for O.D.E. (C.R.C. Press), p. 144.

[24] G. P. Kuiper, in Astrophysics: A Topical Symposium, ed. J. A. Hynek (McGraw-Hill), 1951, p. 357.

[25] A. Morbidelli, Celestial Mechanics and Dynamical Astronomy, 72 (1999) 129.

[26] http://www.newscientist.com/news 7 October 2002. 